\title{
Proofs of two conjectures on Catalan triangle numbers
}

\author{
Victor J. W. Guo and Xiuguo Lian \\ School of Mathematical Sciences, Huaiyin Normal University, Huai'an 223300, Jiangsu, \\ People's Republic of China \\ jwguo@hytc.edu.cn, lianxiuguo@126.com
}

\begin{abstract}
We prove two conjectures on sums of products of Catalan triangle numbers, which were originally conjectured by Miana, Ohtsuka, and Romero [Discrete Math. 340 (2017), 2388-2397]. The first one is proved by using Zeilberger's algorithm, and the second one is proved by establishing its $q$-analogue.

Keywords: Catalan numbers; Catalan triangle; Zeilberger's algorithm; q-Chu-Vandermonde summation; cyclotomic polynomial.

MR Subject Classifications: 05A30; 05A10; 11B65.
\end{abstract}

\section{Introduction}

The well-known Catalan numbers $C_{n}:=\frac{1}{n+1}\left(\begin{array}{c}2 n \\ n\end{array}\right)$ play an important role in combinatorics. For example, the Catalan number $C_{n}$ counts the number of sequences $a_{1}, a_{2}, \ldots, a_{2 n}$ consisting of $n 1$ 's and $n(-1)$ 's such that $a_{1}+\cdots+a_{k} \geqslant 0$ for $k=1, \ldots, 2 n$. See [22, 23, for more combinatorial explanations of the Catalan numbers.

Shapiro [20] introduced the Catalan triangle $\left(B_{n, k}\right)_{n \geqslant k \geqslant 0}$ given by

$$
B_{n, k}:=\frac{k}{n}\left(\begin{array}{c}
2 n \\
n-k
\end{array}\right)=\left(\begin{array}{c}
2 n-1 \\
n-k
\end{array}\right)-\left(\begin{array}{c}
2 n-1 \\
n-k-1
\end{array}\right) .
$$

The Catalan triangle and related topics have been studied by many authors over the past decade. See, for example, [2, 4, 7, 10 13, 16 18, 21, 24, 25]. In particular, Miana, Ohtsuka, and Romero [16, Corollary 3.2(i), Theorem 3.3] proved that

$$
\begin{aligned}
\sum_{k=0}^{n} B_{n, k}^{3} & =\frac{1}{2}\left(\begin{array}{c}
2 n \\
n
\end{array}\right)^{3}-\frac{3}{2}\left(\begin{array}{c}
2 n \\
n
\end{array}\right) \sum_{k=n}^{2 n-1}\left(\begin{array}{l}
k \\
n
\end{array}\right)\left(\begin{array}{c}
k \\
n-1
\end{array}\right) \\
& =\frac{1}{2 n}\left(\begin{array}{c}
2 n \\
n
\end{array}\right) \sum_{k=1}^{n} k\left(\begin{array}{c}
2 n-k-1 \\
n-1
\end{array}\right)^{2}
\end{aligned}
$$

They [16, Conjecture 4.2] made the following conjecture: 
Conjecture 1.1 For all positive integers $m$ and $n$, the identity

$$
\sum_{k=0}^{r} B_{n, k}^{2} B_{m, k}=\frac{1}{2}\left(\begin{array}{c}
2 n \\
n
\end{array}\right)^{2}\left(\begin{array}{c}
2 m \\
m
\end{array}\right)\left[1-\frac{n+2 m}{r}\left(\begin{array}{c}
n+m \\
n
\end{array}\right)^{-1}\left(\begin{array}{c}
n+r \\
n
\end{array}\right)^{-1} \sum_{k=0}^{r-1}\left(\begin{array}{c}
s+k \\
s
\end{array}\right)\left(\begin{array}{c}
n+k \\
n-1
\end{array}\right)\right]
$$

holds for $r=\min \{n, m\}$ and $s=\max \{n, m\}$. In the particular case $m=n$, we recover (1.1).

In this paper, we shall prove the following stronger version of Conjecture 1.1.

Theorem 1.2 The identity (1.2) holds for $r=m$ and $s=n$. It also holds for $r=n$ and $s=m$.

We shall also give the following new identity.

Theorem 1.3 Let $m$ and $n$ be non-negative integers. Then

$$
\sum_{k=1}^{m}\left(\begin{array}{c}
n+k-2 \\
k-1
\end{array}\right)\left(\begin{array}{l}
n-1 \\
k-1
\end{array}\right)\left(\begin{array}{l}
m+n \\
m-k
\end{array}\right)=\sum_{k=0}^{m} k\left(\begin{array}{c}
m+n-k-1 \\
n-1
\end{array}\right)^{2}
$$

Miana, Ohtsuka, and Romero [16] considered the numbers

$$
C_{n, k}:=\frac{n-2 k}{n}\left(\begin{array}{l}
n \\
k
\end{array}\right)=\left(\begin{array}{c}
n-1 \\
k
\end{array}\right)-\left(\begin{array}{c}
n-1 \\
k-1
\end{array}\right)
$$

and called them Catalan triangle numbers. It is easy to see that $B_{n, k}=C_{2 n, n-k}$. Miana, Ohtsuka, and Romero [16, Theorem 1.2(i) and Theorem 3.1(i)] proved that

$$
\begin{aligned}
& \sum_{k=0}^{a} C_{n, k}=\left(\begin{array}{c}
n-1 \\
a
\end{array}\right), \\
& \sum_{k=0}^{a} C_{n, k}^{3}=4\left(\begin{array}{c}
n-1 \\
a
\end{array}\right)^{3}-3\left(\begin{array}{c}
n-1 \\
a
\end{array}\right) \sum_{j=0}^{n-1}\left(\begin{array}{l}
j \\
a
\end{array}\right)\left(\begin{array}{c}
j \\
n-a-1
\end{array}\right),
\end{aligned}
$$

and proposed the following conjecture [16, Conjecture 4.1]:

Conjecture 1.4 Let $n$ and a be positive integers with $n>a$, and let $r$ be a non-negative integer. Then

$$
\sum_{k=0}^{a} C_{n, k}^{2 r+1} \equiv 0 \quad\left(\bmod \left(\begin{array}{c}
n-1 \\
a
\end{array}\right)\right)
$$


Note that Conjecture 1.4 is true for some special cases (see Guo and Zeng [10, Theorem 1.4], Guo and Zeng [11, Theorem 1.4], and Guo and Wang [7, Theorem 1.3]).

It is easy to see that $C_{n, k}=-C_{n, n-k}$. Hence, to prove (1.4), it suffices to prove (1.4) for the case where $n \geqslant 2 a$. It is easy to see that the congruence (1.4) can be written as the following two congruences according to the parity of $n$ (also reversing the summation order):

$$
\begin{gathered}
\sum_{k=a}^{n} B_{n, k}^{2 r+1} \equiv 0 \quad\left(\bmod \left(\begin{array}{c}
2 n-1 \\
n-a
\end{array}\right)\right), \quad 0 \leqslant a \leqslant n, \\
\sum_{k=a}^{n} A_{n, k}^{2 r+1} \equiv 0 \quad\left(\bmod \left(\begin{array}{c}
2 n \\
n-a
\end{array}\right)\right), \quad 0 \leqslant a \leqslant n,
\end{gathered}
$$

where

$$
A_{n, k}:=\frac{2 k+1}{2 n+1}\left(\begin{array}{c}
2 n+1 \\
n-k
\end{array}\right), \quad 0 \leqslant k \leqslant n .
$$

In this paper, we shall confirm Conjecture 1.4 by establishing (1.5) and (1.6), respectively. More precisely, we shall give $q$-analogues of (1.5) and (1.6).

The $q$-integers are defined by $[n]=\frac{1-q^{n}}{1-q}$ and the $q$-shifted factorials (see [5]) are defined by $(a ; q)_{0}=1$ and $(a ; q)_{n}=(1-a)(1-a q) \cdots\left(1-a q^{n-1}\right)$ for $n=1,2, \ldots$ The $q$-binomial coefficients are defined as

$$
\left[\begin{array}{l}
n \\
k
\end{array}\right]=\left[\begin{array}{l}
n \\
k
\end{array}\right]_{q}= \begin{cases}\frac{(q ; q)_{n}}{(q ; q)_{k}(q ; q)_{n-k}}, & \text { if } 0 \leqslant k \leqslant n \\
0, & \text { otherwise. }\end{cases}
$$

It is well-known that the $q$-binomial coefficients are polynomials in $q$ with integer coefficients. The polynomials $B_{n, k}(q)$ and $A_{n, k}(q)$ are given by (see [8, 10])

$$
\begin{aligned}
& B_{n, k}(q):=\frac{[k]}{[n]}\left[\begin{array}{c}
2 n \\
n-k
\end{array}\right], \quad 1 \leqslant k \leqslant n, \\
& A_{n, k}(q):=q^{n-k} \frac{[2 k+1]}{[2 n+1]}\left[\begin{array}{c}
2 n+1 \\
n-k
\end{array}\right]=\left[\begin{array}{c}
2 n \\
n-k
\end{array}\right]-\left[\begin{array}{c}
2 n \\
n-k-1
\end{array}\right], \quad 0 \leqslant k \leqslant n .
\end{aligned}
$$

Let $P(q)$ be a Laurent polynomial in $q$ and let $D(q)$ be a polynomial in $q$. For convenience, we write

$$
P(q) \equiv 0 \quad(\bmod D(q))
$$

if $P(q) / D(q)$ is still a Laurent polynomial in $q$.

We have the following $q$-versions of (1.5) and (1.6).

Theorem 1.5 Let $n$ be a positive integer, and let $a$ and $r$ be non-negative integers with $a \leqslant n$. Then, for $0 \leqslant j \leqslant 2 r+1$, there holds

$$
\sum_{k=a}^{n}\left(1+q^{k}\right) B_{n, k}(q)^{2 r+1} q^{j k^{2}-(r+1) k} \equiv 0 \quad\left(\bmod \left(1+q^{n}\right)\left[\begin{array}{c}
2 n-1 \\
n-a
\end{array}\right]\right) .
$$


Theorem 1.6 Let $n$ be a positive integer, and let $a$ and $r$ be non-negative integers with $a \leqslant n$. Then, for $0 \leqslant j \leqslant 2 r+1$, there holds

$$
\sum_{k=a}^{n} A_{n, k}(q)^{2 r+1} q^{j\left(k^{2}+k\right)} \equiv 0 \quad\left(\bmod \left[\begin{array}{c}
2 n \\
n-a
\end{array}\right]\right) .
$$

It is clear that (1.5) and (1.6) follow from Theorems 1.5 and 1.6 by letting $q=1$. It should also be mentioned that the $a=0$ case of Theorem 1.5 follows from [7, Theorem 1.3], while the $a=0$ case of Theorem [1.6 can be deduced from [8, Theorem 1.3].

\section{Proof of Theorem 1.2}

We first prove (1.2) holds for $r=m$ and $s=n$. Namely,

$$
\sum_{k=0}^{m} B_{n, k}^{2} B_{m, k}=\frac{1}{2}\left(\begin{array}{c}
2 n \\
n
\end{array}\right)^{2}\left(\begin{array}{c}
2 m \\
m
\end{array}\right)\left[1-\frac{n+2 m}{m}\left(\begin{array}{c}
n+m \\
n
\end{array}\right)^{-2} \sum_{k=0}^{m-1}\left(\begin{array}{c}
n+k \\
n
\end{array}\right)\left(\begin{array}{c}
n+k \\
n-1
\end{array}\right)\right] .
$$

It can be easily proved by induction that (see [16, Theorem 2.3(i)])

$$
\left(\begin{array}{c}
n+m \\
n
\end{array}\right)^{2}=\sum_{k=0}^{m} \frac{2 m+n-2 k}{n}\left(\begin{array}{c}
m+n-k-1 \\
n-1
\end{array}\right)^{2}
$$

Moreover, by reversing the summation order, we have

$$
\sum_{k=0}^{m-1}\left(\begin{array}{c}
n+k \\
n
\end{array}\right)\left(\begin{array}{c}
n+k \\
n-1
\end{array}\right)=\sum_{k=0}^{m} \frac{m-k}{n}\left(\begin{array}{c}
m+n-k-1 \\
n-1
\end{array}\right)^{2} .
$$

Hence, the identity (2.1) is equivalent to

$$
2 m\left(\begin{array}{c}
2 n \\
n
\end{array}\right)^{-2}\left(\begin{array}{c}
2 m \\
m
\end{array}\right)^{-1}\left(\begin{array}{c}
n+m \\
n
\end{array}\right)^{2} \sum_{k=0}^{m} B_{n, k}^{2} B_{m, k}=\sum_{k=0}^{m-1} k\left(\begin{array}{c}
m+n-k-1 \\
n-1
\end{array}\right)^{2} .
$$

Denote the left-hand side and right-hand side of (2.2) by $S_{n}(m)$ and $T_{n}(m)$, respectively. Then Zeilberger's algorithm [15, 19] gives

$$
\begin{aligned}
& (2 m+n) S_{n}(m+1)=(2 m+n+2) S_{n}(m)+n\left(\begin{array}{c}
n+m \\
n
\end{array}\right)^{2}, \\
& (2 m+n) T_{n}(m+1)=(2 m+n+2) T_{n}(m)+n\left(\begin{array}{c}
n+m \\
n
\end{array}\right)^{2} .
\end{aligned}
$$

Since $S_{n}(0)=T_{n}(0)=0$, we conclude that $S_{n}(m)=T_{n}(m)$ for all non-negative integers $m$. 
To prove that (1.2) also holds for $r=n$ and $s=m$, noting that

$$
\sum_{k=0}^{m} B_{n, k}^{2} B_{m, k}=\sum_{k=0}^{n} B_{n, k}^{2} B_{m, k}
$$

it suffices to prove

$$
\frac{1}{m}\left(\begin{array}{c}
n+m \\
n
\end{array}\right)^{-1} \sum_{k=0}^{m-1}\left(\begin{array}{c}
n+k \\
n
\end{array}\right)\left(\begin{array}{c}
n+k \\
n-1
\end{array}\right)=\frac{1}{n}\left(\begin{array}{c}
2 n \\
n
\end{array}\right)^{-1} \sum_{k=0}^{n-1}\left(\begin{array}{c}
m+k \\
m
\end{array}\right)\left(\begin{array}{c}
n+k \\
n-1
\end{array}\right)
$$

which is equivalent to

$$
\begin{aligned}
& \frac{m+1}{n}\left(\begin{array}{c}
n+m+1 \\
n
\end{array}\right)\left(\begin{array}{c}
2 n \\
n
\end{array}\right) \sum_{k=0}^{-1}\left(\begin{array}{c}
m+k+1 \\
m+1
\end{array}\right)\left(\begin{array}{c}
n+k \\
n-1
\end{array}\right) \\
& -\frac{m}{n}\left(\begin{array}{c}
n+m \\
n
\end{array}\right)\left(\begin{array}{c}
2 n \\
n
\end{array}\right) \sum_{k=0}^{-1}\left(\begin{array}{c}
m+k \\
m
\end{array}\right)\left(\begin{array}{c}
n+k \\
n-1
\end{array}\right)=\left(\begin{array}{c}
n+m \\
n
\end{array}\right)\left(\begin{array}{c}
n+m \\
n-1
\end{array}\right),
\end{aligned}
$$

or

$$
\sum_{k=0}^{n-1} \frac{(n+k+1) m+(n+1)(k+1)}{m+1}\left(\begin{array}{c}
m+k \\
m
\end{array}\right)\left(\begin{array}{c}
n+k \\
n-1
\end{array}\right)=n\left(\begin{array}{c}
n+m \\
n-1
\end{array}\right)\left(\begin{array}{c}
2 n \\
n
\end{array}\right)
$$

But (2.4) can be easily proved by Gosper's algorithm. This completes the proof of (2.3).

\section{Proof of Theorem 1.3}

The $m=3$ case of $[10$, Corollary 4.1$]$ gives

$$
\begin{aligned}
& \sum_{k=1}^{n_{1}} k^{3}\left(\begin{array}{c}
n_{1}+n_{2} \\
n_{1}+k
\end{array}\right)\left(\begin{array}{c}
n_{2}+n_{3} \\
n_{2}+k
\end{array}\right)\left(\begin{array}{c}
n_{3}+n_{1} \\
n_{3}+k
\end{array}\right) \\
& \quad=\frac{n_{1} n_{3}}{2}\left(\begin{array}{c}
n_{1}+n_{3} \\
n_{1}
\end{array}\right) \sum_{k=1}^{n_{1}}\left(\begin{array}{c}
n_{3}+k-2 \\
k-1
\end{array}\right)\left(\begin{array}{c}
n_{1}-1 \\
k-1
\end{array}\right)\left(\begin{array}{c}
n_{2}+n_{3} \\
n_{2}-k
\end{array}\right) .
\end{aligned}
$$

Namely,

$$
\begin{aligned}
& \sum_{k=1}^{n_{1}} \frac{k^{3}}{n_{1} n_{2} n_{3}}\left(\begin{array}{c}
2 n_{1} \\
n_{1}+k
\end{array}\right)\left(\begin{array}{c}
2 n_{2} \\
n_{2}+k
\end{array}\right)\left(\begin{array}{c}
2 n_{3} \\
n_{3}+k
\end{array}\right) \\
& \quad=\frac{1}{2 n_{2}}\left(\begin{array}{c}
n_{1}+n_{3} \\
n_{1}
\end{array}\right) \frac{\left(n_{1}+n_{2}\right) !\left(n_{2}+n_{3}\right) !\left(n_{3}+n_{1}\right) !}{\left(2 n_{1}\right) !\left(2 n_{2}\right) !\left(2 n_{3}\right) !} \sum_{k=1}^{n_{1}}\left(\begin{array}{c}
n_{3}+k-2 \\
k-1
\end{array}\right)\left(\begin{array}{c}
n_{1}-1 \\
k-1
\end{array}\right)\left(\begin{array}{c}
n_{2}+n_{3} \\
n_{2}-k
\end{array}\right) .
\end{aligned}
$$


Letting $n_{1}=n_{3}=n$ and $n_{2}=m$ in (3.1), we obtain

$$
2 m\left(\begin{array}{c}
2 n \\
n
\end{array}\right)^{-2}\left(\begin{array}{c}
2 m \\
m
\end{array}\right)^{-1}\left(\begin{array}{c}
n+m \\
n
\end{array}\right)^{2} \sum_{k=0}^{m} B_{n, k}^{2} B_{m, k}=\sum_{k=1}^{n}\left(\begin{array}{c}
n+k-2 \\
k-1
\end{array}\right)\left(\begin{array}{l}
n-1 \\
k-1
\end{array}\right)\left(\begin{array}{c}
m+n \\
m-k
\end{array}\right) .
$$

Combining (2.2) and (3.2), we get (1.3).

Remark. The identity (1.3) can also be proved by Zeilberger's algorithm.

\section{Proof of Theorem 1.5}

We first need to establish the following result, which is similar to [7, Theorem 1.1].

Theorem 4.1 Let $n$ be a positive integer and let $a, r$ be non-negative integers with $a \leqslant n$. Then, for $j=0,1$, there holds

$$
\sum_{k=a}^{n}[2 k][k]^{2 r} q^{(r+1)(n-k)+j k^{2}}\left[\begin{array}{c}
2 n \\
n-k
\end{array}\right] \equiv 0 \quad\left(\bmod [n+a]\left[\begin{array}{c}
2 n \\
n-a
\end{array}\right]\right) .
$$

Proof. For $j=0$, let

$$
S_{r}(a, n ; q)=\sum_{k=a}^{n}[2 k][k]^{2 r} q^{(r+1)(n-k)}\left[\begin{array}{c}
2 n \\
n-k
\end{array}\right] .
$$

It is easily seen that (via telescoping)

$$
\begin{aligned}
S_{0}(a, n ; q)=\sum_{k=a}^{n}[2 k] q^{n-k}\left[\begin{array}{c}
2 n \\
n-k
\end{array}\right] & =\sum_{k=a}^{n}[n-k+1]\left[\begin{array}{c}
2 n \\
n-k+1
\end{array}\right]-\sum_{k=a}^{n}[n-k]\left[\begin{array}{c}
2 n \\
n-k
\end{array}\right] \\
& =[n-a+1]\left[\begin{array}{c}
2 n \\
n-a+1
\end{array}\right] \\
& =[n+a]\left[\begin{array}{c}
2 n \\
n-a
\end{array}\right] .
\end{aligned}
$$

For $r \geqslant 1$, since

$$
[k]^{2}\left[\begin{array}{c}
2 n \\
n-k
\end{array}\right] q^{n-k}=[n]^{2}\left[\begin{array}{c}
2 n \\
n-k
\end{array}\right]-[2 n][2 n-1]\left[\begin{array}{c}
2 n-2 \\
n-k-1
\end{array}\right],
$$

we have

$$
S_{r}(a, n ; q)=[n]^{2} S_{r-1}(a, n ; q)-q^{r}[2 n][2 n-1] S_{r-1}(a, n-1 ; q), n=1,2, \ldots
$$


Applying the recurrence relation (4.1) and by induction on $r$, we can easily prove that, for all positive integers $r$, there holds

$$
S_{r}(a, n ; q) \equiv 0 \quad\left(\bmod [n+a]\left[\begin{array}{c}
2 n \\
n-a
\end{array}\right]\right) .
$$

For $j=1$, let

$$
T_{r}(a, n ; q)=\sum_{k=a}^{n}[2 k][k]^{2 r} q^{(r+1)(n-k)+k^{2}}\left[\begin{array}{c}
2 n \\
n-k
\end{array}\right] .
$$

Using the relation $\left[\begin{array}{l}n \\ k\end{array}\right]_{q^{-1}}=\left[\begin{array}{l}n \\ k\end{array}\right] q^{k(k-n)}$, we have $T_{r}(a, n ; q)=q^{n^{2}+2 r n+2 n-2 r-1} S_{r}\left(a, n ; q^{-1}\right)$. Therefore, from (4.2) we deduce that

$$
T_{r}(a, n ; q) \equiv 0 \quad\left(\bmod [n+a]\left[\begin{array}{c}
2 n \\
n-a
\end{array}\right]\right) .
$$

Since both $S_{r}(a, n ; q)$ and $T_{r}(a, n ; q)$ are polynomials in $q$, we obtain the desired result.

We also need the following generalization of Theorem 4.1 .

Theorem 4.2 Let $n_{1}, \ldots, n_{m}, n_{m+1}=n_{1}$ be positive integers. Then for any non-negative integers $a, j$ and $r$ with $a \leqslant n_{1}$ and $j \leqslant m$, the expression

$$
\frac{1}{\left[n_{m}+a\right]}\left[\begin{array}{c}
n_{1}+n_{m} \\
n_{1}-a
\end{array}\right]^{-1} \sum_{k=a}^{n_{1}}[2 k][k]^{2 r} q^{j k^{2}-(r+1) k} \prod_{i=1}^{m}\left[\begin{array}{c}
n_{i}+n_{i+1} \\
n_{i}+k
\end{array}\right]
$$

is a Laurent polynomial in $q$ with integer coefficients.

Proof. Denote (4.3) by $S_{r}\left(a ; n_{1}, \ldots, n_{m} ; j, q\right)$. Let

$$
C\left(a_{1}, \ldots, a_{l} ; k\right)=\prod_{i=1}^{l}\left[\begin{array}{c}
a_{i}+a_{i+1} \\
a_{i}+k
\end{array}\right] \quad\left(a_{l+1}=a_{1}\right) .
$$

Then we can write $S_{a}\left(n_{1}, \ldots, n_{m} ; r, j, q\right)$ as

$$
S_{r}\left(a ; n_{1}, \ldots, n_{m} ; j, q\right)=\frac{(q ; q)_{n_{1}-a}(q ; q)_{n_{m}+a-1}}{(q ; q)_{n_{1}+n_{m}}} \sum_{k=a}^{n_{1}}[2 k][k]^{2 r} q^{j k^{2}-(r+1) k} C\left(n_{1}, \ldots, n_{m} ; k\right) .
$$

For $m \geqslant 3$, there holds

$$
C\left(n_{1}, \ldots, n_{m} ; k\right)=\frac{(q ; q)_{n_{2}+n_{3}}(q ; q)_{n_{m}+n_{1}}}{(q ; q)_{n_{1}+n_{2}}(q ; q)_{n_{m}+n_{3}}}\left[\begin{array}{c}
n_{1}+n_{2} \\
n_{1}+k
\end{array}\right]\left[\begin{array}{c}
n_{1}+n_{2} \\
n_{2}+k
\end{array}\right] C\left(n_{3}, \ldots, n_{m} ; k\right) .
$$

By the $q$-Chu-Vandermonde summation formula (see, for example, [1, p. 37, (3.3.10)]), we have

$$
\left[\begin{array}{c}
n_{1}+n_{2} \\
n_{1}+k
\end{array}\right]=\sum_{s=0}^{n_{1}-k}\left[\begin{array}{c}
n_{1}-k \\
s
\end{array}\right]\left[\begin{array}{c}
n_{2}+k \\
s+2 k
\end{array}\right] q^{s(s+2 k)}
$$


which may be rewritten as

$$
\left[\begin{array}{c}
n_{1}+n_{2} \\
n_{1}+k
\end{array}\right]\left[\begin{array}{c}
n_{1}+n_{2} \\
n_{2}+k
\end{array}\right]=\sum_{s=0}^{n_{1}-k} \frac{q^{s^{2}+2 k s}(q ; q)_{n_{1}+n_{2}}}{(q ; q)_{s}(q ; q)_{s+2 k}(q ; q)_{n_{1}-k-s}(q ; q)_{n_{2}-k-s}}
$$

where we assume that $\frac{1}{(q ; q)_{n}}=0$ for any negative integer $n$. Substituting (4.5) and (4.6) into (4.4), we obtain

$$
\begin{aligned}
& S_{r}\left(a ; n_{1}, \ldots, n_{m} ; j, q\right) \\
& =\frac{(q ; q)_{n_{2}+n_{3}}(q ; q)_{n_{1}-a}(q ; q)_{n_{m}+a-1}}{(q ; q)_{n_{m}+n_{3}}} \sum_{k=a}^{n_{1}} \sum_{s=0}^{n_{1}-k} \frac{q^{s^{2}+2 k s+j k^{2}-(r+1) k}[2 k][k]^{2 r} C\left(n_{3}, \ldots, n_{m} ; k\right)}{(q ; q)_{s}(q ; q)_{s+2 k}(q ; q)_{n_{1}-k-s}(q ; q)_{n_{2}-k-s}} \\
& \quad=\frac{(q ; q)_{n_{2}+n_{3}}(q ; q)_{n_{1}-a}(q ; q)_{n_{m}+a-1}}{(q ; q)_{n_{m}+n_{3}}} \sum_{l=a}^{n_{1}} q^{l^{2}} \sum_{k=a}^{l} \frac{q^{(j-1) k^{2}-(r+1) k}[2 k][k]^{2 r} C\left(n_{3}, \ldots, n_{m} ; k\right)}{(q ; q)_{l-k}(q ; q)_{l+k}(q ; q)_{n_{1}-l}(q ; q)_{n_{2}-l}}
\end{aligned}
$$

where $l=s+k$. Noticing that, for $m \geqslant 3$,

$$
\frac{C\left(n_{3}, \ldots, n_{m} ; k\right)}{(q ; q)_{l-k}(q ; q)_{l+k}}=\frac{(q ; q)_{n_{m}+n_{3}}}{(q ; q)_{n_{3}+l}(q ; q)_{n_{m}+l}} C\left(l, n_{3}, \ldots, n_{m} ; k\right)
$$

we are led to the following recurrence relation

$$
S_{r}\left(a ; n_{1}, \ldots, n_{m} ; j, q\right)=\sum_{l=a}^{n_{1}} q^{l^{2}}\left[\begin{array}{c}
n_{1}-a \\
l-a
\end{array}\right]\left[\begin{array}{c}
n_{2}+n_{3} \\
n_{2}-l
\end{array}\right] S_{r}\left(a ; l, n_{3}, \ldots, n_{m} ; j-1, q\right) .
$$

Similarly, for $m=2$, we have

$$
S_{r}\left(a ; n_{1}, n_{2} ; j, q\right)=\sum_{l=a}^{n_{1}} q^{l^{2}}\left[\begin{array}{c}
n_{1}-a \\
l-a
\end{array}\right]\left[\begin{array}{c}
n_{2}+a-1 \\
l+a-1
\end{array}\right] S_{r}(a ; l ; j-1, q) .
$$

Now we can give an inductive proof of the theorem. For $m=1$, the conclusion is true by Theorem 4.1. Suppose that $S_{r}\left(a ; n_{1}, \ldots, n_{m-1} ; j, q\right)$ is a Laurent polynomial in $q$ with integer coefficients for some $m \geqslant 2$ and all $j$ with $0 \leqslant j \leqslant m-1$. Then by (4.7) (if $m \geqslant 3$ ) or (4.8) (if $m=2)$, so is $S_{r}\left(a ; n_{1}, \ldots, n_{m} ; j, q\right)$ for $1 \leqslant j \leqslant m$. Furthermore, since

$$
S_{r}\left(a ; n_{1}, \ldots, n_{m} ; 0, q\right)=S_{r}\left(a ; n_{1}, \ldots, n_{m} ; m, q^{-1}\right) q^{n_{1} n_{2}+n_{2} n_{3}+\cdots+n_{m-1} n_{m}-n_{m}+a\left(a+n_{m}-n_{1}-1\right)-2 r}
$$

for $m \geqslant 2$, one sees that $S_{r}\left(a ; n_{1}, \ldots, n_{m} ; 0, q\right)$ is also a Laurent polynomial in $q$ with integer coefficients. This completes the proof.

Note that, by (4.8) and the $q$-Chu-Vandermonde summation formula, there holds

$$
S_{0}\left(a ; n_{1}, n_{2} ; 1, q\right)=\left[\begin{array}{c}
n_{1}+n_{2}-1 \\
n_{1}+a-1
\end{array}\right] q^{a^{2}-a} .
$$

Let $\Phi_{n}(x)$ be the $n$-th cyclotomic polynomial. The following result is very useful in dealing with $q$-binomial coefficients (see [14, (10)] or [3, 9]). 
Proposition 4.3 The q-binomial coefficient $\left[\begin{array}{c}m \\ k\end{array}\right]$ can be factorized into

$$
\left[\begin{array}{c}
m \\
k
\end{array}\right]=\prod_{d} \Phi_{d}(q)
$$

where the product is over all positive integers $d \leqslant m$ such that $\lfloor k / d\rfloor+\lfloor(m-k) / d\rfloor<$ $\lfloor m / d\rfloor$.

Proof of Theorem 1.5. Letting $m=2 r+1$ and $n_{1}=\cdots=n_{2 r+1}=n$ in Theorem 4.2, we see that

$$
\begin{aligned}
& \frac{1}{[n+a]}\left[\begin{array}{c}
2 n \\
n-a
\end{array}\right]^{-1} \sum_{k=a}^{n}[2 k][k]^{2 r} q^{j k^{2}-(r+1) k}\left[\begin{array}{c}
2 n \\
n-k
\end{array}\right]^{2 r+1} \\
& =\frac{1}{[2 n]}\left[\begin{array}{c}
2 n-1 \\
n-a
\end{array}\right]^{-1} \sum_{k=a}^{n}\left(1+q^{k}\right) q^{j k^{2}-(r+1) k}\left([n] B_{n, k}(q)\right)^{2 r+1}
\end{aligned}
$$

is a Laurent polynomial in $q$ with integer coefficients. Note that $B_{n, k}(q)$ is a polynomial in $q$ with integer coefficients (see [6, 10]). Therefore,

$$
\frac{\operatorname{gcd}\left([2 n]\left[\begin{array}{c}
2 n-1 \\
n-a
\end{array}\right],[n]^{2 r+1}\right)}{[2 n]\left[\begin{array}{c}
2 n-1 \\
n-a
\end{array}\right]} \sum_{k=a}^{n}\left(1+q^{k}\right) q^{j k^{2}-(r+1) k} B_{n, k}(q)^{2 r+1}
$$

is a Laurent polynomial in $q$ with integer coefficients.

It is well-known that

$$
[n]=\prod_{\substack{d \mid n \\ d>1}} \Phi_{d}(q)
$$

Therefore, from Proposition 4.3 we immediately deduce that

$$
\operatorname{gcd}\left(\left[\begin{array}{c}
2 n-1 \\
n-a
\end{array}\right],[n]\right)=1
$$

Moreover, we have $[2 n]=\left(1+q^{n}\right)[n]$ and $\operatorname{gcd}\left(1+q^{n},[n]\right)=1$. It follows that

$$
\frac{\operatorname{gcd}\left([2 n]\left[\begin{array}{c}
2 n-1 \\
n-a
\end{array}\right],[n]^{2 r+1}\right)}{[2 n]\left[\begin{array}{c}
2 n-1 \\
n-a
\end{array}\right]}=\frac{1}{\left(1+q^{n}\right)\left[\begin{array}{c}
2 n-1 \\
n-a
\end{array}\right]} \text {. }
$$

This completes the proof. 


\section{Proof of Theorem 1.6}

We first need to establish the following result.

Lemma 5.1 Let $n$ be a positive integer and let $a, s$ be non-negative integers with $a \leqslant n$. Then

$$
\sum_{k=a}^{n} q^{n-k}[2 k+1]\left[\begin{array}{c}
2 n+1 \\
n-k
\end{array}\right]\left(q^{-k} ; q\right)_{s}\left(q^{k+1} ; q\right)_{s} \equiv 0 \quad\left(\bmod [2 n+1]\left[\begin{array}{c}
2 n \\
n-a
\end{array}\right]\right) .
$$

Proof. We proceed by induction on $s$. For $s=0$, we have

$$
\begin{aligned}
\sum_{k=a}^{n} q^{n-k}[2 k+1]\left[\begin{array}{c}
2 n+1 \\
n-k
\end{array}\right] & =[2 n+1] \sum_{k=a}^{n}\left(\left[\begin{array}{c}
2 n \\
n-k
\end{array}\right]-\left[\begin{array}{c}
2 n \\
n-k-1
\end{array}\right]\right) \\
& =[2 n+1]\left[\begin{array}{c}
2 n \\
n-a
\end{array}\right] .
\end{aligned}
$$

Suppose (5.1) is true for $s$. Noticing the relations

$$
\begin{aligned}
& {\left[\begin{array}{c}
2 n+1 \\
n-k
\end{array}\right]\left(q^{-k} ; q\right)_{s+1}\left(q^{k+1} ; q\right)_{s+1}} \\
& =\left(1-q^{s-n}\right)\left(1-q^{s+n+1}\right)\left[\begin{array}{c}
2 n+1 \\
n-k
\end{array}\right]\left(q^{-k} ; q\right)_{s}\left(q^{k+1} ; q\right)_{s} \\
& \quad+q^{s-n}\left(1-q^{2 n}\right)\left(1-q^{2 n+1}\right)\left[\begin{array}{c}
2 n-1 \\
n-k-1
\end{array}\right]\left(q^{-k} ; q\right)_{s}\left(q^{k+1} ; q\right)_{s}
\end{aligned}
$$

and

$$
[2 n][2 n+1][2 n-1]\left[\begin{array}{c}
2 n-2 \\
n-a-1
\end{array}\right]=[2 n+1]\left[\begin{array}{c}
2 n \\
n-a
\end{array}\right][n-a][n+a],
$$

we can easily prove that (5.1) holds for $s+1$.

We have the following generalization of (5.1).

Lemma 5.2 Let $n$ be a positive integer and let $a, r, s$ be non-negative integers with $a \leqslant n$. Then

$$
\sum_{k=a}^{n} q^{-(2 r+1) k}[2 k+1]^{2 r+1}\left[\begin{array}{c}
2 n+1 \\
n-k
\end{array}\right]\left(q^{-k} ; q\right)_{s}\left(q^{k+1} ; q\right)_{s} \equiv 0 \quad\left(\bmod [2 n+1]\left[\begin{array}{c}
2 n \\
n-a
\end{array}\right]\right) .
$$

Proof. We proceed by induction on $r$. Denote the left-hand side of (5.3) by $X_{r}(a, n, s ; q)$. By (5.1), one sees that (5.3) is true for $r=0$. For $r \geqslant 1$, suppose that

$$
X_{r-1}(a, n, s ; q) \equiv 0 \quad \bmod [2 n+1]\left[\begin{array}{c}
2 n \\
n-a
\end{array}\right]
$$


holds for any positive integer $n$ and non-negative integers $a, s$ with $a \leqslant n$. It is easy to check that

$$
\begin{aligned}
{\left[\begin{array}{c}
2 n+1 \\
n-k
\end{array}\right][2 k+1]^{2}=} & q^{2 k-2 n}\left[\begin{array}{c}
2 n+1 \\
n-k
\end{array}\right][2 n+1]^{2} \\
& -q^{2 k-2 n}\left[\begin{array}{c}
2 n-1 \\
n-k-1
\end{array}\right][2 n][2 n+1]\left(1+q^{n-s}\right)\left(1+q^{n+s+1}\right) \\
& +q^{2 k-n-s}\left[\begin{array}{c}
2 n-1 \\
n-k-1
\end{array}\right][2 n][2 n+1]\left(1-q^{s-k}\right)\left(1-q^{s+k+1}\right),
\end{aligned}
$$

and therefore,

$$
\begin{aligned}
X_{r}(a, n, s ; q)= & q^{-2 n}[2 n+1]^{2} X_{r-1}(a, n, s ; q) \\
& -q^{-2 n}[2 n][2 n+1]\left(1+q^{n-s}\right)\left(1+q^{n+s+1}\right) X_{r-1}(a, n-1, s ; q) \\
& +q^{-n-s}[2 n][2 n+1] X_{r-1}(a, n-1, s+1 ; q) .
\end{aligned}
$$

By the induction hypothesis and applying (5.2), we immediately deduce from the recurrence (5.4) that (5.3) holds for $r$.

Theorem 5.3 Let $n_{1}, \ldots, n_{m}, n_{m+1}=n_{1}$ be positive integers. Then for any non-negative integers $a, j$ and $r$ with $a \leqslant n_{1}$ and $j \leqslant m$, the expression

$$
\frac{1}{\left[n_{1}+n_{m}+1\right]}\left[\begin{array}{c}
n_{1}+n_{m} \\
n_{1}-a
\end{array}\right]^{-1} \sum_{k=a}^{n_{1}} q^{j\left(k^{2}+k\right)-(2 r+1) k}[2 k+1]^{2 r+1} \prod_{i=1}^{m}\left[\begin{array}{c}
n_{i}+n_{i+1}+1 \\
n_{i}-k
\end{array}\right]
$$

is a Laurent polynomial in $q$ with integer coefficients.

Proof. Denote (5.5) by $\bar{S}_{r}\left(a ; n_{1}, \ldots, n_{m} ; j, q\right)$. Let

$$
\bar{C}\left(a_{1}, \ldots, a_{l} ; k\right)=\prod_{i=1}^{l}\left[\begin{array}{c}
a_{i}+a_{i+1}+1 \\
a_{i}-k
\end{array}\right],
$$

where $a_{l+1}=a_{1}$. Then

$$
\begin{aligned}
& \bar{S}_{r}\left(a ; n_{1}, \ldots, n_{m} ; j, q\right) \\
& \quad=\frac{(q ; q)_{n_{1}-a}(q ; q)_{n_{m}+a}}{(q ; q)_{n_{1}+n_{m}+1}} \sum_{k=a}^{n_{1}} q^{j\left(k^{2}+k\right)-(2 r+1) k}[2 k+1]^{2 r+1} \bar{C}\left(n_{1}, \ldots, n_{m} ; k\right),
\end{aligned}
$$

For $m \geqslant 3$, we have

$$
\bar{C}\left(n_{1}, \ldots, n_{m} ; k\right)=\frac{(q ; q)_{n_{2}+n_{3}+1}(q ; q)_{n_{m}+n_{1}+1}}{(q ; q)_{n_{1}+k+1}(q ; q)_{n_{2}-k}(q ; q)_{n_{m}+n_{3}+1}}\left[\begin{array}{c}
n_{1}+n_{2}+1 \\
n_{1}-k
\end{array}\right] \bar{C}\left(n_{3}, \ldots, n_{m} ; k\right) .
$$


Applying (5.7) and the $q$-Chu-Vandermonde summation formula (see [1, p. 37, (3.3.10)])

$$
\left[\begin{array}{c}
n_{1}+n_{2}+1 \\
n_{1}-k
\end{array}\right]=\sum_{s=0}^{n_{1}-k} \frac{q^{s(s+2 k+1)}(q ; q)_{n_{1}+k+1}(q ; q)_{n_{2}-k}}{(q ; q)_{s}(q ; q)_{s+2 k+1}(q ; q)_{n_{1}-k-s}(q ; q)_{n_{2}-k-s}}
$$

we may write (5.6) as

$$
\begin{aligned}
& \bar{S}_{r}\left(a ; n_{1}, \ldots, n_{m} ; j, q\right) \\
& =\frac{(q ; q)_{n_{2}+n_{3}+1}(q ; q)_{n_{1}-a}(q ; q)_{n_{m}+a}}{(q ; q)_{n_{m}+n_{3}+1}} \\
& \quad \times \sum_{k=a}^{n_{1}} \sum_{s=0}^{n_{1}-k} \frac{q^{j\left(k^{2}+k\right)-(2 r+1) k+s(s+2 k+1)}[2 k+1]^{2 r+1} \bar{C}\left(n_{3}, \ldots, n_{m} ; k\right)}{(q ; q)_{s}(q ; q)_{s+2 k+1}(q ; q)_{n_{1}-k-s}(q ; q)_{n_{2}-k-s}} \\
& =\frac{(q ; q)_{n_{2}+n_{3}+1}(q ; q)_{n_{1}-a}(q ; q)_{n_{m}+a}}{(q ; q)_{n_{m}+n_{3}+1}} \\
& \quad \times \sum_{l=a}^{n_{1}} q^{l^{2}+l} \sum_{k=a}^{l} \frac{q^{(j-1)\left(k^{2}+k\right)-(2 r+1) k}[2 k+1]^{2 r+1} \bar{C}\left(n_{3}, \ldots, n_{m} ; k\right)}{(q ; q)_{l-k}(q ; q)_{l+k}(q ; q)_{n_{1}-l}(q ; q)_{n_{2}-l}}
\end{aligned}
$$

where $l=s+k$. Noticing that

$$
\frac{\bar{C}\left(n_{3}, \ldots, n_{m} ; k\right)}{(q ; q)_{l-k}(q ; q)_{l+k+1}}=\frac{(q ; q)_{n_{m}+n_{3}+1}}{(q ; q)_{n_{3}+l+1}(q ; q)_{n_{m}+l+1}} \bar{C}\left(l, n_{3}, \ldots, n_{m} ; k\right)
$$

we obtain

$$
\bar{S}_{r}\left(a ; n_{1}, \ldots, n_{m} ; j, q\right)=\sum_{l=a}^{n_{1}} q^{l^{2}+l}\left[\begin{array}{c}
n_{1}-a \\
l-a
\end{array}\right]\left[\begin{array}{c}
n_{2}+n_{3}+1 \\
n_{2}-l
\end{array}\right] \bar{S}_{r}\left(a ; l, n_{3}, \ldots, n_{m} ; j-1, q\right)
$$

for $m \geqslant 3$. Similarly, for $m=2$, applying (5.8) we get

$$
\bar{S}_{r}\left(a ; n_{1}, n_{2} ; j, q\right)=\sum_{l=a}^{n_{1}} q^{l^{2}+l}\left[\begin{array}{c}
n_{1}-a \\
l-a
\end{array}\right]\left[\begin{array}{c}
n_{2}+a \\
l+a
\end{array}\right] \bar{S}_{r}(a ; l ; j-1, q) .
$$

Similarly to the inductive proof of Theorem 4.2, using (5.3) (with $s=0$ ), (5.9), (5.10) and the following relation

$$
\bar{S}_{r}\left(a ; n_{1}, \ldots, n_{m} ; 0, q\right)=\bar{S}_{r}\left(a ; n_{1}, \ldots, n_{m} ; m, q^{-1}\right) q^{n_{1} n_{2}+n_{2} n_{3}+\cdots+n_{m-1} n_{m}-n_{1}-n_{m}+a\left(a+n_{m}-n_{1}\right)}
$$

for $m \geqslant 2$, we can prove the theorem.

Note that, by (5.10) and the $q$-Chu-Vandermonde summation formula, there holds

$$
\bar{S}_{0}\left(a ; n_{1}, n_{2} ; 1, q\right)=\left[\begin{array}{c}
n_{1}+n_{2} \\
n_{1}+a
\end{array}\right] q^{a^{2}} .
$$


Proof of Theorem 1.6. Letting $m=2 r+1$ and $n_{1}=\cdots=n_{2 r+1}=n$ in Theorem 5.3, we see that

$$
\begin{aligned}
& \frac{1}{[2 n+1]}\left[\begin{array}{c}
2 n \\
n-a
\end{array}\right]^{-1} \sum_{k=a}^{n} q^{j\left(k^{2}+k\right)-(2 r+1) k}[2 k+1]^{2 r+1}\left[\begin{array}{c}
2 n+1 \\
n-k
\end{array}\right]^{2 r+1} \\
& =\frac{1}{[2 n+1]}\left[\begin{array}{c}
2 n \\
n-a
\end{array}\right]^{-1} \sum_{k=a}^{n} q^{j\left(k^{2}+k\right)-(2 r+1) n}[2 n+1]^{2 r+1} A_{n, k}(q)^{2 r+1}
\end{aligned}
$$

is a Laurent polynomial in $q$ with integer coefficients. By (1.7), we know that $A_{n, k}(q)$ is a polynomial in $q$ with integer coefficients. It follows that

$$
\frac{\operatorname{gcd}\left([2 n+1]\left[\begin{array}{c}
2 n \\
n-a
\end{array}\right],[2 n+1]^{2 r+1}\right)}{[2 n+1]\left[\begin{array}{c}
2 n \\
n-a
\end{array}\right]} \sum_{k=a}^{n} A_{n, k}(q)^{2 r+1} q^{j\left(k^{2}+k\right)}
$$

is a Laurent polynomial in $q$ with integer coefficients. By (4.9) and Proposition 4.3, it is easy to see that

$$
\operatorname{gcd}\left(\left[\begin{array}{c}
2 n \\
n-a
\end{array}\right],[2 n+1]\right)=1
$$

and so

$$
\frac{\operatorname{gcd}\left([2 n+1]\left[\begin{array}{c}
2 n \\
n-a
\end{array}\right],[2 n+1]^{2 r+1}\right)}{[2 n+1]\left[\begin{array}{c}
2 n \\
n-a
\end{array}\right]}=\frac{1}{\left[\begin{array}{c}
2 n \\
n-a
\end{array}\right]} .
$$

This completes the proof.

\section{Two open problems}

In this section, we give two related conjectures for further study. The first one is a stronger version of Theorem [4.2, and is also a generalization of [7, Conjecture 6.3].

Conjecture 6.1 Let $n_{1}, \ldots, n_{m}, n_{m+1}=n_{1}$ be positive integers. Then, for any integer $j$ and non-negative integers $a, r$ with $a \leqslant n_{1}$, the expression

$$
\frac{1}{\left[n_{m}+a\right]}\left[\begin{array}{c}
n_{1}+n_{m} \\
n_{1}-a
\end{array}\right]^{-1} \sum_{k=a}^{n_{1}}[2 k][k]^{2 r} q^{j k^{2}-(r+1) k} \prod_{i=1}^{m}\left[\begin{array}{c}
n_{i}+n_{i+1} \\
n_{i}+k
\end{array}\right]
$$

is a Laurent polynomial in $q$, and it has non-negative integer coefficients if $0 \leqslant j \leqslant m$.

Conjecture 6.2 Theorem 5.3 is still true for any integer $j$.

Acknowledgments. The authors thank the anonymous referee for a careful reading of this paper. The first author was partially supported by the National Natural Science Foundation of China (grant 11771175), the Natural Science Foundation of Jiangsu Province (grant BK20161304), and the Qing Lan Project of Education Committee of Jiangsu Province. 


\section{References}

[1] G.E. Andrews, The Theory of Partitions, Cambridge University Press, Cambridge, 1998.

[2] X. Chen, W. Chu, Moments on Catalan numbers, J. Math. Anal. Appl. 349 (2009), 311-316.

[3] W.Y.C. Chen and Q.-H. Hou, Factors of the Gaussian coefficients, Discrete Math. 306 (2006), 1446-1449.

[4] W. Chu, Moments on quadratic binomial products, J. Number Theory 178 (2017), 19-30.

[5] G. Gasper and M. Rahman, Basic Hypergeometric Series, Second Edition, Encyclopedia of Mathematics and Its Applications, Vol. 96, Cambridge University Press, Cambridge, 2004.

[6] V.J.W. Guo and C. Krattenthaler, Some divisibility properties of binomial and $q$-binomial coefficients, J. Number Theory, 135 (2013), 167-184.

[7] V.J.W. Guo, S.-D. Wang, Factors of sums involving $q$-binomial coefficients and powers of $q$-integers, J. Diff. Equ. Appl. 23 (2017), 1670-1679.

[8] V.J.W. Guo, S.-D. Wang, Factors of sums and alternating sums of products of $q$-binomial coefficients and powers of $q$-integers, Taiwanese J. Math., doi: 10.11650/tjm/180601.

[9] V.J.W. Guo, J. Zeng, Some arithmetic properties of the $q$-Euler numbers and $q$-Salié, numbers, European J. Combin. 27 (2006), 884-895.

[10] V.J.W. Guo, J. Zeng, Factors of binomial sums from the Catalan triangle, J. Number Theory 130 (2010), 172-186.

[11] V.J.W. Guo, J. Zeng, Factors of sums and alternating sums involving binomial coefficients and powers of integers, Int. J. Number Theory 7 (2011), 1959-1976.

[12] J.M. Gutiérrez, M.A. Hernández, P.J. Miana, N. Romero, New identities in the Catalan triangle, J. Math. Anal. Appl. 341 (2008), 52-61.

[13] E. Kilic, H. Prodinger, Identities with squares of binomial coefficients: an elementary and explicit approach, Publ. Inst. Math. 99 (113) (2016), 243-248.

[14] D. Knuth and H. Wilf, The power of a prime that divides a generalized binomial coefficient, J. Reine Angew. Math. 396 (1989), 212-219.

[15] W. Koepf, Hypergeometric Summation-An Algorithmic Approach to Summation and Special Function Identities, 2nd Ed., Springer, London, 2014.

[16] P.J. Miana, H. Ohtsuka, N. Romero, Sums of powers of Catalan triangle numbers, Discrete Math. 340 (2017), 2388-2397.

[17] P.J. Miana, N. Romero, Computer proofs of new identities in the Catalan triangle, in: Proc. of the "Segundas Jornadas de Teoría de Números", Madrid, 2007, Biblioteca de la Revista Matemática Iberoamericana, pp. 203-208.

[18] P.J. Miana, N. Romero, Moments of combinatorial and Catalan numbers, J. Number Theory 130 (2010), 1876-1887.

[19] M. Petkovšek, H.S. Wilf, and D. Zeilberger, $A=B$, A K Peters, Ltd., Wellesley, MA, 1996.

[20] L.W. Shapiro, A Catalan triangle, Discrete Math. 14 (1976), 83-90.

[21] S. Stanimirović, P. Stanimirović, A. Ilić, Ballot matrix as Catalan matrix power and related identities, Discrete Appl. Math. 160 (2012), 344-351.

[22] R.P. Stanley, Enumerative Combinatorics, Vol. 2, Cambridge University Press, Cambridge, 1999. 
[23] R.P. Stanley, Catalan Numbers, Cambridge University Press, Cambridge, 2015.

[24] Y. Sun, F. Ma, Some new binomial sums related to the Catalan triangle, Electron. J. Combin. 21 (1) (2014) \#P1.33.

[25] Z. Zhang, B. Pang, Several identities in the Catalan triangle, Indian J. Pure Appl. Math. 41 (2010), 363-378. 